\title{
AUTOMATED OPTIMIZATION OF RESIDUAL REDUCTION ALGORITHM PARAMETERS IN
} OPENSIM

\author{
Jordan T. Sturdy ${ }^{1}$, Anne K. Silverman ${ }^{1}$, Nathan T. Pickle ${ }^{1,2}$ \\ ${ }^{1}$ Colorado School of Mines, Golden, CO \\ ${ }^{2}$ CFD Research Corp., Huntsville, AL
}

Word count: Abstract (124), Paper (1995)

Keywords: Biomechanics, Dynamic Consistency, OpenSim, RRA, MATLAB, Python, movement simulation 


\section{Abstract}

The residual reduction algorithm (RRA) in OpenSim improves dynamic consistency of movement simulations of musculoskeletal models. RRA requires the user to select numerous tracking weights for the joint kinematics to reduce residual errors. Selection is often performed manually, which can be time-consuming and is unlikely to yield optimal tracking weights. A multi-heuristic optimization algorithm was employed to expedite tracking weight decision making to reduce residual errors. This method produced more rigorous results than manual iterations and although the total computation time was not significantly reduced, this method does not require the user to monitor the algorithm's progress to find a solution, thereby reducing manual tuning. Supporting documentation and code to implement this optimization is freely provided to assist the community with developing movement simulations.

\section{INTRODUCTION}

Musculoskeletal models and simulations have seen significant increase in adoption in recent years, facilitated by software platforms such as OpenSim (Delp et al., 2007, simtk.org). Inverse dynamics calculations are frequently the basis of these simulations. The inverse dynamics problem takes experimental observations, i.e., ground reaction forces and joint kinematics, and calculates the joint moments that produced the experimentally observed data (Yamaguchi, 2005). This calculation contains multiple sources of error including modeling assumptions (e.g., rigid body assumptions, segment inertial properties), and measurement error (e.g., soft tissue artifact). These sources of error result in dynamic inconsistency (Kingma and Toussaint, 1996; Pearsall and Costigan, 1999; Riemer et al., 2008) and produce fictitious forces in the simulated motion. Several methods have been proposed to address this issue (Cahouët et al., 2002; Ganley and Powers, 2004; Kuo, 1998), including OpenSim's residual reduction algorithm (RRA, https://simtkconfluence.stanford.edu/display/OpenSim/Residual+Reduction+Algorithm) that minimizes the fictitious, or "residual", forces and moments applied at the pelvis. RRA first computes changes to the total model mass as $\Delta m=F_{y, a v g} / g$, where $F_{y, a v g}$ is the average vertical residual force across the simulation and $g$ is acceleration due to gravity. RRA also calculates an altered torso center of mass (COM) location. In addition, RRA can change the model joint accelerations slightly to achieve higher dynamic consistency and further reduce residual forces and moments. Thus, there is a tradeoff between the residuals and the kinematic errors, where the tradeoff is governed by user-selected tracking weights. OpenSim's documentation recommends users manually adjust the tracking weights until the residuals are minimized, which is a time-consuming process. In this paper we describe an open-source software tool (provided in Python and MATLAB) for optimization of tracking weights in RRA, which provides biomechanics researchers with an automated method for selecting RRA tracking weights, increasing efficiency in developing high-quality movement simulations.

\section{METHODS}

A Tracking Weight Selection Algorithm (TWSA) was developed to optimize the tracking weight values, which serve as inputs to the optimization algorithm RRA in OpenSim. A modified random hill climbing algorithm was used to explore the search space. 


\subsection{OBJECTIVE FUNCTION FORMULATION}

The TWSA minimized the sum of root-mean-squared (RMS) residual forces and moments and the sum of RMS kinematic tracking errors as a weighted multi-objective cost function:

$$
\min W_{R} \sum_{i=1}^{m}\left(w_{i} R_{i}\right)^{p_{R}}+W_{E} \sum_{j=1}^{n}\left(w_{j} E_{j}\right)^{p_{E}}
$$

where $R_{i}$ is the RMS of a residual term, $W_{R}$ and $W_{E}$ are the user-specified weights applied to the sums of the forces and errors, $w_{j}$ and $w_{i}$ are the weights applied to individual errors and forces, $E_{j}$ is the RMS of a particular error, $p_{R}$ and $p_{E}$ are user specified powers and $m$ and $n$ are the number of residuals and errors examined respectively. Each RMS value is normalized by recommended maximum values that are provided by OpenSim ${ }^{1}$ by applying the weights, $w_{j}$ and $w_{i}$. The variables, $p_{R}$ and $p_{E}$, are applied to the residuals and errors to work effectively as barrier functions. They penalize each RMS value as the value increases above the recommended max values.

\subsection{ALGORITHM DESCRIPTION}

The TWSA minimized the residual forces and moments while tracking the experimental data. A set of uniform tracking weights was used to perform the initial RRA and the objective function was evaluated in a MATLAB function and reported to the TWSA. This first evaluation of the objective function was the current best solution. An array of parameters, $\tau$, was then created to perturb tracking weights. Each element in $\tau$ was calculated as $\tau_{i}=b^{t}$, where $b$ started at 1.5 and was lowered to 1.1 after $75 \%$ of the TWSA iterations were performed, and $t$ was an integer ranging from -2 to 2 selected from a random sample which was weighted to favor negative values (decrease the tracking weight) if coordinate tracking was below a good threshold, positive values if tracking was bad (increase the tracking weight), and equal chances otherwise. Each tracking weight was then multiplied by the corresponding $\tau_{i}$ to generate a new set of tracking tasks, which were used to run the next RRA iteration. The objective function was evaluated after each iteration, and the tracking weights corresponding to the current lowest objective function value were used as the starting weights that are perturbed for subsequent iterations. This was repeated until an iteration limit set by the user was reached. The resulting solutions from the TWSA do not guarantee optimality but are better than the original starting point, meaning that the solutions have lower residual forces and moments while still maintaining acceptable kinematic tracking errors (Hicks et al., 2015).

\subsection{TEST CASE}

To test the effectiveness of the TWSA, we selected a single participant from publicly available data repository (simtk.org/home/nmbl_running, Hamner \& Delp, 2013) for a common dynamic task: running. Three running strides each were isolated from motion files of 2.0, 3.0, 4.0, and $5.0 \mathrm{~m} / \mathrm{s}$, and were used to develop the running simulations. The TWSA was applied using two different models. First, the full body model developed by Hamner et al. (Hamner et al., 2010; Hamner and Delp, 2013) was used because it was consistent with the dataset. Next, the gait2392 model (Delp et al., 2007) was selected because it is widely used 
and available with OpenSim installation. A key distinction between these models is that gait2392 uses a lumped head/arms/trunk

segment, whereas the Hamner2010 model implements multi-segment arms. This difference in model formulation can be used to

demonstrate the robustness of the TWSA, as ignoring contributions of the arms during running leads to dynamic inconsistency in the

simulation. Prior to the TWSA, RRA was run iteratively using fixed tracking weights, and recommended model mass adjustments

were performed until the total mass change was less than $0.001 \mathrm{~kg}$. Then, the TWSA was applied to each running trial using the mass

adjusted model. For this study, 200 RRA iterations were performed by the TWSA, and the tracking weights associated with the best

objective function value were then re-run to produce the optimal RRA results. Residuals after the TWSA were compared to residuals

prior to the TWSA to determine simulation improvement. The peak net external force from each running trial was used to normalize

residuals for evaluation (Hicks et al., 2015), and both peak and RMS residuals were analyzed. Tracking errors were evaluated to

ensure all coordinates were within measurement error.

\section{RESULTS}

Mass changes were less than $0.5 \mathrm{~kg}$ for all trials using both the Hamner2010 model and the gait2392 model. Torso center of mass

changes were less than $6 \mathrm{~cm}$ for most trials, and adjustments were slightly larger for the Hamner2010 model (Table 1.). Triall in the 2.0

$\mathrm{m} / \mathrm{s}$ condition required the largest change in center of mass location, greater than $10 \mathrm{~cm}$, using both models.

Table 1. Changes made to the torso center of mass for each trial using both the Hamner2010 and gait2392 models. Values are reported in $\mathrm{cm}$ as $(\mathrm{x}, \mathrm{z})$ pairs for the anterior $(\mathrm{x})$ and medio-lateral $(\mathrm{z})$ directions.

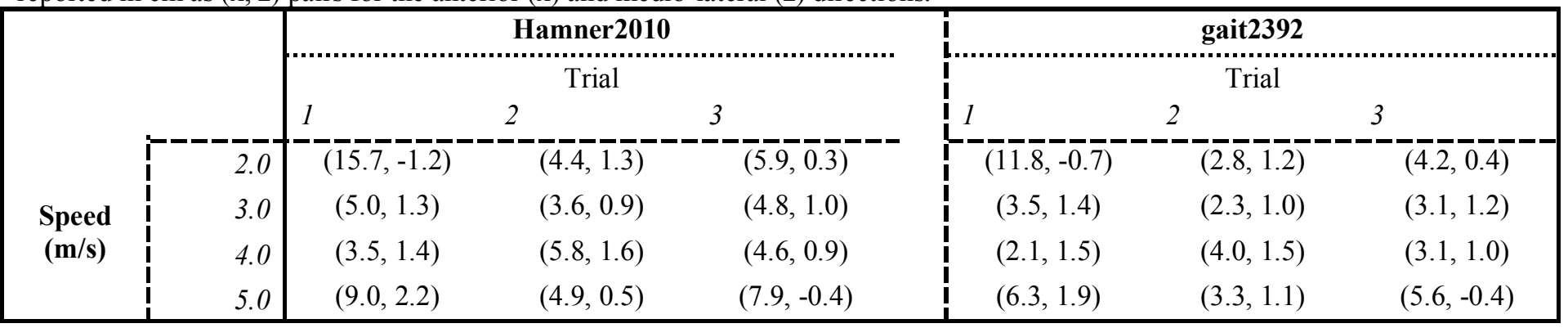

We ran the TWSA on a $3.60 \mathrm{GHz}$ CPU with 12 logical processors used to optimize tracking weights, with each running speed condition $(2.0,3.0,4.0$, and $5.0 \mathrm{~m} / \mathrm{s})$ evaluated in parallel. The 200 iterations took the most time to complete during the $2 \mathrm{~m} / \mathrm{s}$ condition, finishing in 2 hours and 4 minutes using the Hamner2010 model, and 49 minutes using the gait2392 model. TWSA objective function values were substantially reduced in the first 50 RRA iterations and achieved reasonable convergence between 100 and 150 iterations (Fig. 1). However, gradual reductions continue to occur with more iterations, and the lowest objective function values were produced in the last 50 iterations for all trials. Using both the Hamner2010 and gait2392 models, the TWSA produced objective function values less than 2.5 on average for $3,4 \mathrm{~m} / \mathrm{s}$ trials. Average best objective function values were 8.99 and 5.84 at 2 and $5 \mathrm{~m} / \mathrm{s}$, respectively, using the Hamner2010 model and were 5.28 and 8.26 using the gait2392 model at 2 and $5 \mathrm{~m} / \mathrm{s}$, respectively. Trial 1 at $2 \mathrm{~m} / \mathrm{s}$ had the highest 
77 objective function value for both models, but the $2 \mathrm{~m} / \mathrm{s}$ trials otherwise achieved values less than 1.1. In addition, certain trials

78 sporadically produced peaks in objective function values between 50 and 150 iterations of the TWSA, and these were most pronounced

99 using the Hamner2010 model (Fig. 1.).

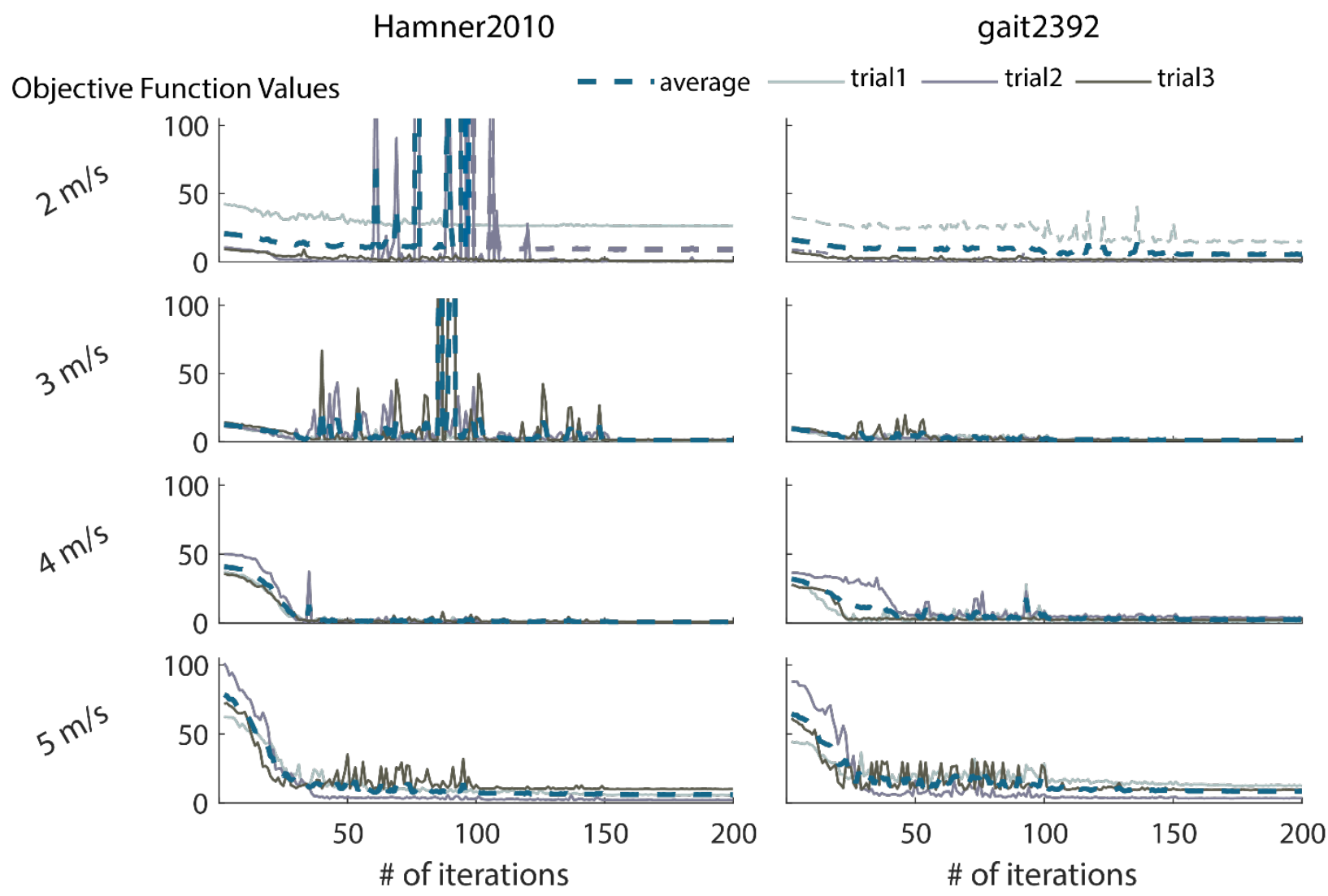

Figure 1. Objective function values for the tracking weight selection algorithm optimization at each RRA iteration using the Hamner2010 model (left) and gait2392 model (right). Three trials were averaged at each iteration for both models at all running speeds (blue dashed lines).

0

After tracking weight optimization, peak and RMS residuals were substantially reduced except for the FZ residual (Fig. 2). RMS forces were less than $1 \%$ peak external force and RMS moments were less than $0.35 \%$ peak external force for both models after tracking weight optimization when averaged across speeds, with improvement in all residuals except FZ (Table 2.). Residuals were slightly larger using the gait2392 model compared to the Hamner2010 model after tracking weight selection. In addition, the FY residual was improved more across all speeds when using the Hamner2010 model compared to gait2392 achieving both a lower peak (Fig 2.) and RMS value (Table 2). 
bioRxiv preprint doi: https://doi.org/10.1101/2021.10.06.463431; this version posted October 8, 2021. The copyright holder for this preprint (which was not certified by peer review) is the author/funder, who has granted bioRxiv a license to display the preprint in perpetuity. It is made available under aCC-BY 4.0 International license.
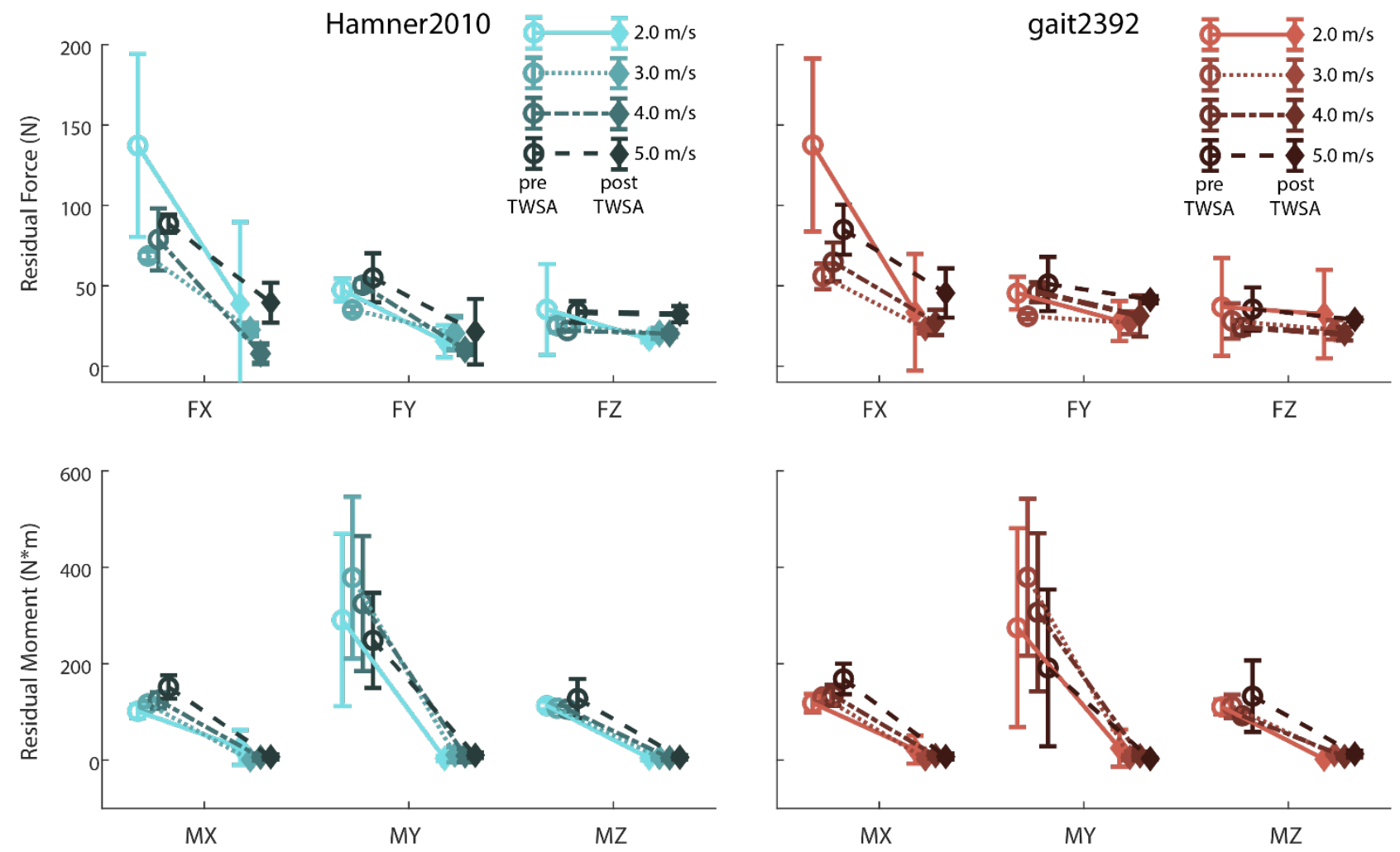

Figure 2. Peak residual forces (top) and moments (bottom) averaged at each running speed and plotted before (pre) and after (post) the tracking weight selection algorithm was applied. Error bars represent the standard deviation of three trials at each speed using the Hamner2010 (left) and gait2392 (right) models.

Table 2. Root mean squared (RMS) residual forces and moments normalized to OpenSim recommended maximum values (Hicks et al., 2015). RMS forces and moments are normalized to $5 \%$ and $1 \%$ respectively of the peak external force (net ground reaction force) during each trial. Values reported are the average RMS of 3 trials for each running speed both before (Pre TWSA) and after (Post TWSA) the tracking weight optimization using both models. Values above 1 indicate the residual exceeds recommended thresholds.

\begin{tabular}{|c|c|c|c|c|c|c|c|c|c|c|c|c|c|}
\hline & \multicolumn{6}{|c|}{ Hamner 2010} & \multicolumn{6}{|c|}{ gait2392 } \\
\hline & & $F X$ & $F Y$ & $F Z$ & $M X$ & $M Y$ & $M Z$ & $F X$ & $F Y$ & $F Z$ & $M X$ & $M Y$ & $M Z$ \\
\hline \multirow{4}{*}{$\begin{array}{c}\text { Pre } \\
\text { TWSA }\end{array}$} & $2.0 \mathrm{~m} / \mathrm{s}$ & 0.44 & 0.27 & 0.13 & 2.66 & 3.84 & 3.45 & 0.40 & 0.24 & 0.11 & 3.30 & 2.98 & 3.29 \\
\hline & $3.0 \mathrm{~m} / \mathrm{s}$ & 0.31 & 0.18 & 0.11 & 2.45 & 4.67 & 2.48 & 0.26 & 0.16 & 0.08 & 2.89 & 3.61 & 2.55 \\
\hline & $4.0 \mathrm{~m} / \mathrm{s}$ & 0.41 & 0.20 & 0.14 & 3.03 & 4.78 & 2.42 & 0.35 & 0.19 & 0.08 & 3.19 & 2.91 & 2.30 \\
\hline & $5.0 \mathrm{~m} / \mathrm{s}$ & 0.52 & 0.25 & 0.18 & 3.30 & 6.00 & 3.01 & 0.46 & 0.23 & 0.13 & 3.58. & 2.94 & 3.01 \\
\hline \multirow{4}{*}{$\begin{array}{c}\text { Post } \\
\text { TWSA }\end{array}$} & $2.0 \mathrm{~m} / \mathrm{s}$ & 0.24 & 0.11 & 0.11 & 0.80 & 0.11 & 0.17 & 0.22 & 0.18 & 0.09 & 0.64 & 0.87 & 0.06 \\
\hline & $3.0 \mathrm{~m} / \mathrm{s}$ & 0.12 & 0.11 & 0.12 & 0.02 & 0.29 & 0.15 & 0.13 & 0.14 & 0.08 & 0.07 & 0.17 & 0.37 \\
\hline & $4.0 \mathrm{~m} / \mathrm{s}$ & 0.04 & 0.07 & 0.15 & 0.21 & 0.24 & 0.16 & 0.15 & 0.16 & 0.08 & 0.27 & 0.26 & 0.24 \\
\hline & $5.0 \mathrm{~m} / \mathrm{s}$ & 0.23 & 0.13 & 0.17 & 0.19 & 0.32 & 0.19 & 0.26 & 0.24 & 0.12 & 0.17 & 0.08 & 0.42 \\
\hline
\end{tabular}




\section{DISCUSSION}

The TWSA performed well using two different musculoskeletal models and improved residual forces and moments by similar amounts with each model. We performed 200 iterations of RRA within the TWSA, but this upper limit may not be necessary to obtain comparable improvements in residuals. This upper limit can also be adjusted by the user. Other than for one trial in both 2 and $5 \mathrm{~m} / \mathrm{s}$ using the gait2392 model, the best objective function value was improved by less than 1 in the last 100 RRA iterations. While more iterations will guarantee a broader search of the solution space, a "good enough" objective function value could be used as a threshold

to stop the TWSA regardless of the number of iterations performed. An objective value threshold implementation would allow researchers to use more computational time on trials with higher residuals, like the first $2 \mathrm{~m} / \mathrm{s}$ trial in this study.

Residual moments were substantially improved in all directions in both models by the TWSA, while improvements in residual forces differed between models and directions. Some of this discrepancy between residuals may be attributed to the objective function formulation. The recommended normalization for residuals (Hicks et al., 2015) is stricter for moments than for forces, and thus, the forces without introducing excessive kinematic tracking errors. The residual forces and moments are trivial to reduce if the kinematics are not tracked, which results in very low residuals but very high errors. Thus, the TWSA sought to simultaneously minimize the residuals and minimize kinematic error. Balancing both of these two objectives is desired.

Other algorithms have been used to automate RRA tracking weight selection using similar objective functions (e.g. Samaan,

Weinhandl, Bawab, \& Ringleb, 2016), and many different optimization approaches could be successfully applied to this application.

While it is challenging to directly the TWSA to others because different motions and data were used, our approach yielded similar kinematic accuracy (see Appendix) and meaningful residual improvement with less computational time compared to other approaches.

\section{CONCLUSIONS}

The TWSA provides a valuable new tool for efficiently computing optimal RRA tracking weights. This algorithm is an important and practical contribution to the modeling and simulation community and may be widely used to improve the quality of musculoskeletal 
bioRxiv preprint doi: https://doi.org/10.1101/2021.10.06.463431; this version posted October 8,2021 . The copyright holder for this preprint (which was not certified by peer review) is the author/funder, who has granted bioRxiv a license to display the preprint in perpetuity. It is made available under aCC-BY 4.0 International license.

H1 amount of time $(<2 \mathrm{hr})$, requiring little or no user input. Supporting documentation and code to implement the TWSA in both Python

and MATLAB are available for download at: https://github.com/FxnlBiomechLab/rra-optimization.

\section{ACKNOWLEDGEMENTS}

The authors would like to thank Daniel Anderson and Moriah Hunt for their contributions to the development of the initial TWSA algorithm. The authors also acknowledge Amy Hegarty and Alexandra Newman for testing of the tool.

\section{REFERENCES}

Cahouët, V., Luc, M., David, A., 2002. Static optimal estimation of joint accelerations for inverse dynamics problem solution. J. Biomech. 35, 1507-13.

Delp, S.L., Anderson, F.C., Arnold, A.S., Loan, P., Habib, A., John, C.T., Guendelman, E., Thelen, D.G., 2007. OpenSim: Opensource software to create and analyze dynamic simulations of movement. IEEE Trans. Biomed. Eng. 54, 1940-1950. https://doi.org/10.1109/TBME.2007.901024

Ganley, K.J., Powers, C.M., 2004. Determination of lower extremity anthropometric parameters using dual energy X-ray absorptiometry: the influence on net joint moments during gait. Clin. Biomech. 19, 50-56. https://doi.org/10.1016/j.clinbiomech.2003.08.002

Hamner, S.R., Delp, S.L., 2013. Muscle contributions to fore-aft and vertical body mass center accelerations over a range of running speeds. J. Biomech. 46, 780-787. https://doi.org/10.1016/j.jbiomech.2012.11.024

Hamner, S.R., Seth, A., Delp, S.L., 2010. Muscle contributions to propulsion and support during running. J. Biomech. 43, $2709-2716$. https://doi.org/10.1016/j.jbiomech.2010.06.025

Hicks, J.L., Uchida, T.K., Seth, A., Rajagopal, A., Delp, S.L., 2015. Is my model good enough? Best practices for verification and validation of musculoskeletal models and simulations of movement. J. Biomech. Eng. 137, 020905-1-020905-24. https://doi.org/10.1115/1.4029304

Kingma, I., Toussaint, H., 1996. Segment inertial parameter evaluation in two anthropmetric models by application of a dynamic linked segment model. J. Biomech. 29, 693-704.

Kuo, A.D., 1998. A least-squares estimation approach to improving the precision of inverse dynamics computations. J. Biomech. Eng. $120,148-59$.

Pearsall, D.J., Costigan, P. a, 1999. The effect of segment parameter error on gait analysis results. Gait Posture 9, 173-83.

Riemer, R., Hsiao-Wecksler, E.T., Zhang, X., 2008. Uncertainties in inverse dynamics solutions: a comprehensive analysis and an application to gait. Gait Posture 27, 578-88. https://doi.org/10.1016/j.gaitpost.2007.07.012

Samaan, M.A., Weinhandl, J.T., Bawab, S.Y., Ringleb, S.I., 2016. Determining residual reduction algorithm kinematic tracking weights for a sidestep cut via numerical optimization. Comput. Methods Biomech. Biomed. Engin. 19, 1721-1729. https://doi.org/10.1080/10255842.2016.1183123

Yamaguchi, G.T., 2005. Dynamic Modeling of Musculoskeletal Motion: A Vectorized Approach for Biomechanical Analysis in Three Dimensions. Springer. 
bioRxiv preprint doi: https://doi.org/10.1101/2021.10.06.463431; this version posted October 8,2021 . The copyright holder for this preprint (which was not certified by peer review) is the author/funder, who has granted bioRxiv a license to display the preprint in perpetuity. It is made available under aCC-BY 4.0 International license.

Table A2. Largest root mean squared tracking error for each coordinate across all running speeds and trials using the gait2392 model.

Table A1. Largest root mean squared tracking error for each coordinate across all running speeds and trials using the Hamner2010 model.

Hamner2010

\begin{tabular}{|c|c|c|c|c|c|c|c|}
\hline Translational & & pelvis_tx & pelvis_ty & pelvis_tz & & & \\
\hline$(\mathrm{cm})$ & Pelvis & 0.92 & 1.14 & 0.97 & & & \\
\hline \multirow{11}{*}{$\begin{array}{l}\text { Rotational } \\
\quad \text { (deg) }\end{array}$} & \multirow{3}{*}{ Pelvis } & pelvis_tilt & pelvis_list & pelvis_rotation & & & \\
\hline & & 0.54 & 1.24 & 1.96 & & & \\
\hline & & lumbar_extension & lumbar_bending & lumbar_rotation & & & \\
\hline & \multirow[t]{2}{*}{ Torso } & 1.61 & 2.28 & 2.16 & & & \\
\hline & & hip_flexion & hip_adduction & hip_rotation & knee_angle & ankle_angle & \\
\hline & \multirow[t]{2}{*}{ Right leg } & 1.99 & 1.19 & 0.19 & 0.85 & 0.19 & \\
\hline & & hip_flexion & hip_adduction & hip_rotation & knee_angle & ankle_angle & \\
\hline & \multirow[t]{2}{*}{ Left leg } & 1.10 & 1.08 & 0.76 & 0.30 & 0.17 & \\
\hline & & arm_flex & arm_add & arm_rot & elbow_flex & pro_sup & \\
\hline & \multirow[t]{2}{*}{ Right arm } & 0.38 & 0.80 & 0.13 & 0.53 & 0.15 & \\
\hline & & arm_flex & arm_add & arm_rot & elbow_flex & pro_sup & \\
\hline
\end{tabular}

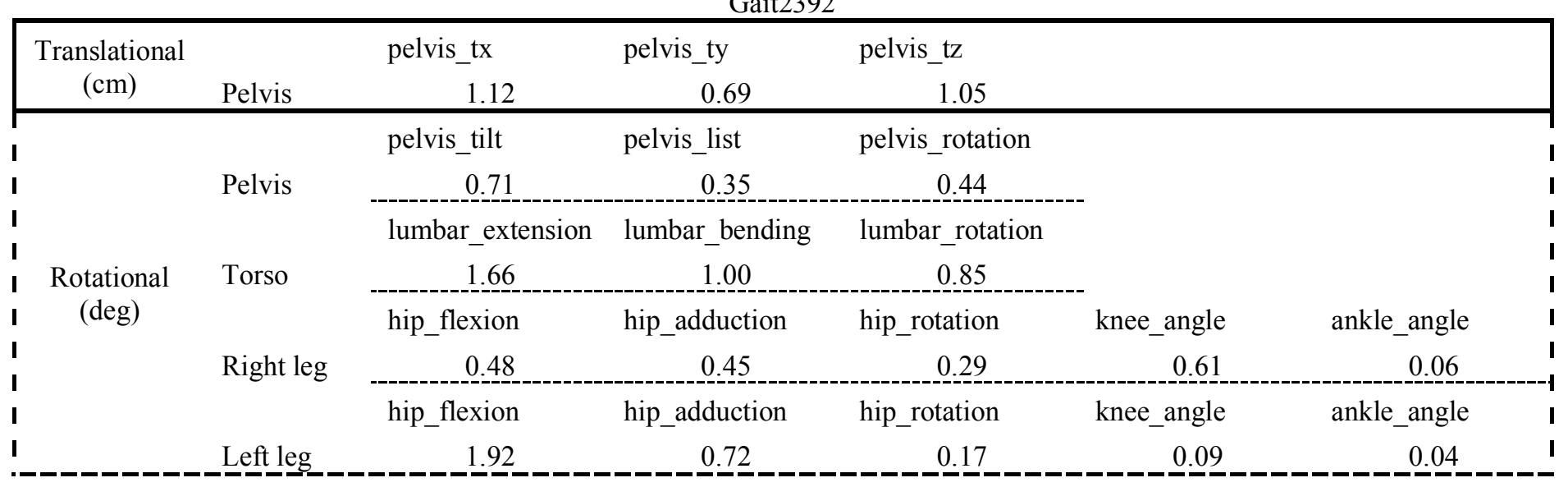

